\title{
Schwartz Jampel syndrome responding positively to carbamazepine therapy: a case report and a novel mutation
}

\author{
Gürkan Gürbüz $z^{1}$ Hatice Mutlu Albayrak² \\ Departments of ${ }^{1}$ Pediatric Neurology and ${ }^{2}$ Pediatric Genetics, Cengiz Gökçek Maternity and Children's Hospital, Gaziantep, \\ Turkey.E-mail:drgurkangurbuz@hotmail.com \\ Received: 26th January 2019, Accepted: 9th March 2019
}

SUMMARY: Gürbüz G, Mutlu Albayrak H. Schwartz Jampel syndrome responding positively to carbamazepine therapy: a case report and a novel mutation. Turk J Pediatr 2019; 61: 967-970.

Schwartz Jampel syndrome was first described in 1962. It is an autosomal recessive disease with generalized myotonic myopathy and skeletal dysplasia. A mutation in the HSPG2 gene occurs. Approximately 150 cases have been reported in literature. A 4-year-old girl presented to the pediatric neurology clinic due to difficulty in walking. The patient had difficulty opening her mouth and swallowing. She was unable to eat solid foods and was bottle fed. She was able to stand leaning forward, with her legs open and with one hand supported. Bilateral blepharospasm, posterior cleft palate, microstomia, pursed lips, kyphoscoliosis, contracture in the elbows, long thin fingers and campodactyly in the bilateral 5th fingers were present. Myotonic contraction with thenar percussion was observed. Previously undescribed mutation was determined in HSPG2 gene in the genetic study. Oral carbamazepine therapy was initiated and 1.5 months later the patient's muscle rigidity had decreased and her motor skills had improved. This report contributes to the literature by defining a new mutation in HSPG2 gene and showing the importance of early diagnosis of the disease.

Key words: carbamazepine, myotonia, Schwartz Jampel syndrome.

Schwartz-Jampel syndrome (SJS), also known as chondrodystrophic myotonia, is an autosomal recessive disease progressing with generalized myotonic myopathy and skeletal dysplasia. We report a case of SJS presenting due to diffuse muscle rigidity and difficulty in walking and swallowing and with typical findings such as blepharospasm, pursed lips, a discontented facial expression and severe skeletal dysplasia. This case is reported due to its rarity and to the detection of a previously undescribed novel mutation.

\section{Case Report}

A 4-year-old girl presented to the pediatric neurology clinic due to difficulty in walking.
She was born by normal vaginal delivery, weighing $2500 \mathrm{~g}$. Her parents were first-degree cousins, and there were no similar cases in the family. She had begun walking with difficulty, with support, at the age of 2.5 years, but became quickly fatigued, and had been unable to stand up for the previous one year. The patient had difficulty opening her mouth and swallowing. She was unable to eat solid foods and was bottle fed. At examination her height, body weight and head circumference were below the 3 rd percentile. She was able to stand leaning forward, with her legs open and with one hand supported. Bilateral blepharospasm, posterior cleft palate, microstomia, pursed lips, kyphoscoliosis, contracture in the elbows, long thin fingers and campodactyly in the 
bilateral 5th fingers were present (Figs 1-4). She was able to clench her hands, but had difficulty opening them. Myotonic contraction with thenar percussion was observed. Deep tendon reflexes could not be elicited. The patient attempted to stand in a crouch gait. Continuous muscle activity at rest, and highfrequency spontaneous repetitive potentials were observed on electromyography (EMG). SJS was suspected on the basis of all these clinical findings. The HSPG2 gene responsible for the disease was sent for mutation analysis, and previously undescribed IVS24A $>$ g (c.318+A>G) mutation was determined.

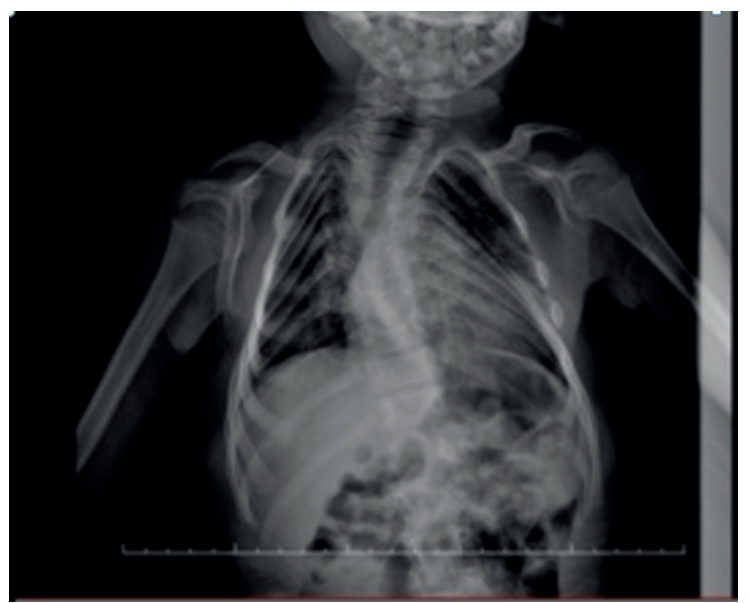

Fig. 1. Posterior -anterior chest X-ray of the patient shows prominent kyphoscoliosis, narrow thoracic outlet.

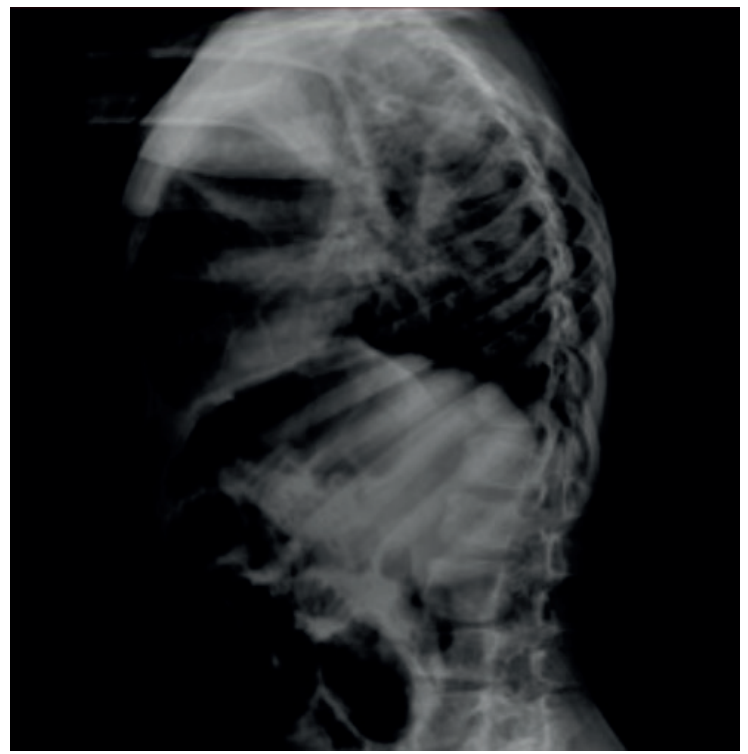

Fig. 2. Note the narrow tharacic outlet and irregularities on vertabral surfaces.
Oral carbamazepine $(20 \mathrm{mg} / \mathrm{kg} /$ day $)$ therapy was initiated as an anti-myotonic agent for the myotonia and muscle rigidity, together with physiotherapy. At follow-up 1.5 months subsequently, the patient's muscle rigidity had decreased and her motor skills had improved. However, there was no change in swallowing

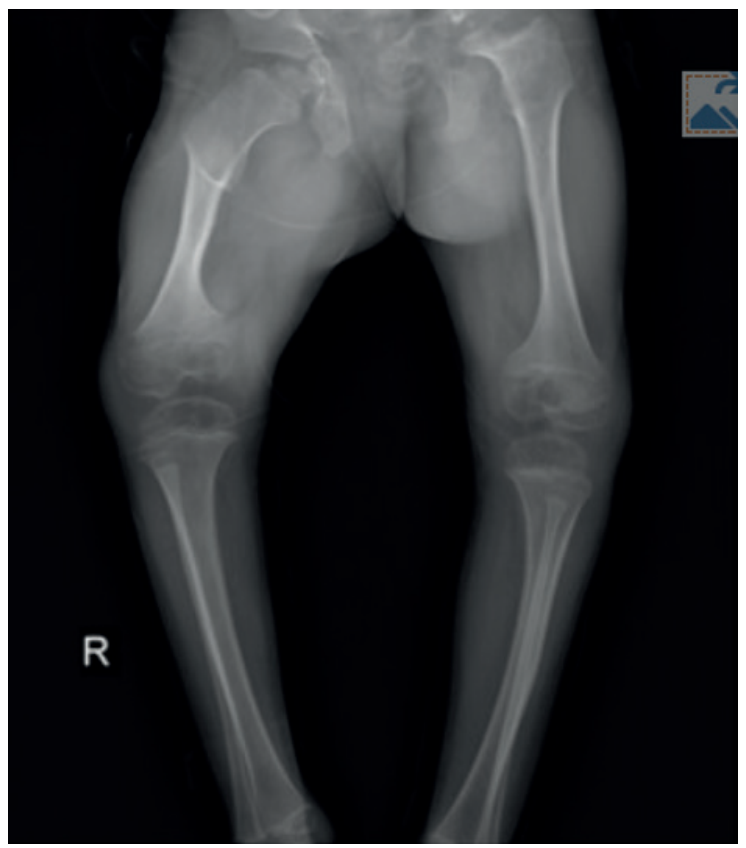

Fig. 3. Presence of short right femur, irregularities of the left femoral head, anterior bowing of the right tibia and widened epiphyses.
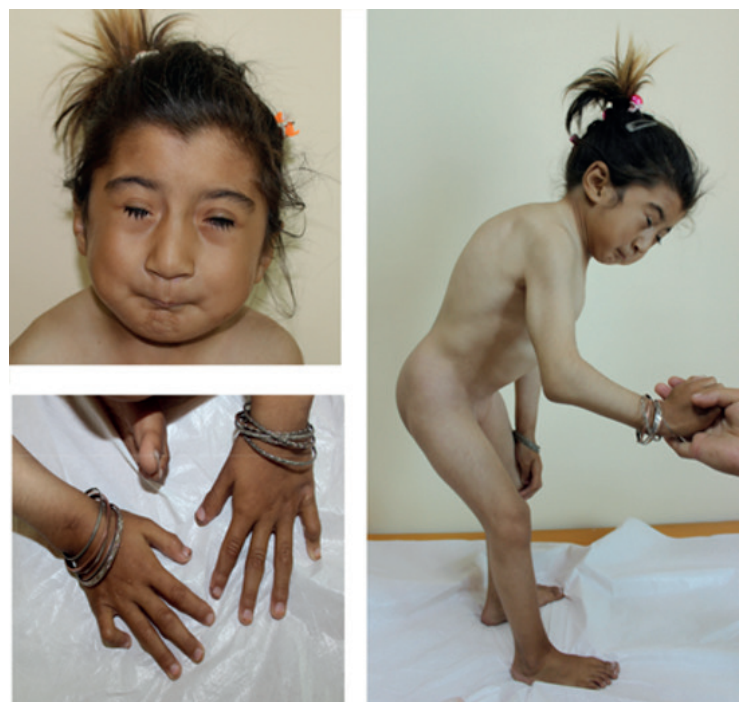

Fig. 4. The patient has bilateral blepharospasm, microstomia, pursed lips, kyphoscoliosis, contracture in the elbows, long thin fingers and campodactyly in the bilate ral 5th fingers and these are typical features for Schwartz Jampel syndrome. 
and chewing function. Due to the potentially life-threatening complications that may emerge with anesthesia and to the disease being transmitted in an autosomal recessive manner, the family was informed that the condition might occur in $25 \%$ of future children. Informed consent was received from the family.

\section{Discussion}

SJS was first described in 1962 in a paper titled "Congenital blepharophimosis associated with unique generalized myopathy." The disease is also known as chondrodystrophic myotonia. Typical phenotypic findings include short stature, a mask-like face, a short neck, bilateral blepharospasm, microstomia, pursed lips, kyphoscoliosis, articular contractures and low-set ears. Vertebral, epiphyseal and metaphyseal chondrodysplasias may also be seen. $^{2}$

There are two forms of SJS, type 1 and type 2. Type 1 has two subforms, $1 \mathrm{~A}$ and 1B. Type $1 \mathrm{~A}$ is the most common and classic form. It is frequently diagnosed in childhood, and myotonia and bone dysplasia are not very severe. Type 1B is associated with diffuse bone dysplasia at birth and maldevelopment of the vertebral column. Myotonia may emerge subsequently. Type 2 is the most severe form, and patients frequently die in the neonatal period. $^{3}$

Myotonia may be observed clinically and at electrophysiology. Typical myotonic muscle contracture is observed after repeated movements such as hand opening and closing or with thenar muscle percussion. Continuous muscle activity and highfrequency spontaneous repetitive potentials are seen at needle EMG. ${ }^{4}$ Laboratory tests are not generally diagnostic, and moderate increased in creatine kinase and aldolases have been determined in some cases. Muscle biopsy shows non-specific changes. ${ }^{5}$ Spinal $\mathrm{x}$-ray is useful in showing kyphoscoliosis and other skeletal anomalies.

Mutation in the heparan sulfate proteoglycan 2 (HSPG2) gene, that encodes Perlecan, in the chromosome 1p34-36.1 has been determined in genetic studies. ${ }^{6}$ Perlecan is one of the principal components of the basal membrane and produces hyperexcitability. Mutations in this gene result in abnormal ion channel expression and impair acetylcholine esterase aggregation. ${ }^{7}$ Perlecan also occurs in cartilage and bone marrow stromal cells and plays a role in cartilage development and bone repair. A defect in Perlecan production thus results in chondrodysplasia and pseudofractures. ${ }^{8}$ A case of SJS with gastrointestinal bleeding associated with vascular structure impairment due to the presence of Perlecan in the vascular endothelium. ${ }^{9}$ These patients are at great risk of fatal hyperthermia during anesthesia, and it is important for patients to be made aware of this. ${ }^{10}$

Treatment is symptomatic. Potential benefits have been reported from ion channel regulating anti-myotonic agents such as mexiletine, procainamide and carbamazepine, employed in the current study. ${ }^{11}$ Physiotherapy, warming, and stretching may also be beneficial. Botulinum toxin administration and levator palpebrae resection may be alternatives for blepharospasm. ${ }^{12}$

Approximately 130 cases have been reported in the literature. The publication of these cases in international journals will be useful in terms of establishing clinical-genetic correlation and increasing the recognition of the syndrome.

Myotonia in our patient improved with carbamazepine. For now, short-term effects have been observed in increased motor skill acquisition, but long-term follow-up is planned. Early identification of these patients is essential in order to prevent fatal complications such as malignant hyperthermia, and early initiation of antimyotonic agents and physiotherapy is important in terms of preventing motor losses.

\section{Acknowledgement}

Genetic analysis was studied in Intergen Genetic Diseases and Diagnosis Centre by Dr. Serdar Ceylaner.

\section{REFERENCES}

1. Schwartz O, Jampel RS. Congenital blepharophimosis associated with a unique generalized myopathy. Arch Ophthalmol 1963; 68: 52-57. 
2. Wiedemann HR, Stuve A. Stuve-Wiedemann syndrome: Update and historical footnote. Am J Med Genet 1996; 63: 12-16.

3. Giedion A, Boltshauser E, Briner J, et al. Heterogeneity in Schwartz-Jampel chondrodystrophic myotonia. Eur J Pediatr 1997; 156: 214-223.

4. Viljoen D, Beighton P. Schwartz-Jampel syndrome (chondrodystrophic myotonia) J Med Genet 1992; 29: $58-62$.

5. Ho NC, Sandusky S, Madike V, Francomano CA, Dalakas MC. Clinico-pathogenetic findings and management of chondrodystrophic myotonia (Schwartz-Jampel syndrome): a case report. BMC Neurol 2003; 3: 3.

6. Arikawa-Hirasawa E, Le AH, Nishino I, et al. Structural and functional mutations of the perlecan gene cause Schwartz-Jampel syndrome, with myotonic myopathy and chondrodysplasia. Am J Hum Genet 2002; 70 : 1368-1375.

7. Peng HB, Xie H, Rossi SG, Rotundo RL. Acetylcholinesterase clustering at the neuromuscular junction involves perlecan and dystroglycan. J Cell Biol 1999; 145: 911-921.
8. Nicole S, Davoine CS, Topaloglu H, et al. Perlecan, the major proteoglycan of basement membranes, is altered in patients with Schwartz-Jampel syndrome (chondrodystrophic myotonia). Nat Genet 2000; 26: 480-483.

9. Polat İ, Karaoğlu P, Yiş U, Kurul SH. Schwartz-Jampel syndrome with gastroduodenal bleeding. J Pediatr Neurosci 2016; 11: 255-257.

10. Ray S, Rubin AP. Anaesthesia in a child with SchwartzJampel syndrome. Anaesthesia 1994; 49: 600-602.

11. Topaloglu H, Serdaroglu A, Okan M, Gucuyener $\mathrm{K}$, Topcu M. Improvement of myotonia with carbamazepine in three cases with the SchwartzJampel syndrome. Neuropediatrics 1993; 24: 232234.

12. Lucci LM, Yen MT, Anderson RL, Hwang IP, Black RE. Orbicularis myectomy with levator advancement in Schwartz-Jampel syndrome. Am J Ophthalmol 2001; 132: 799-801. 